http://jmscr.igmpublication.org/home/ ISSN (e)-2347-176x ISSN (p) 2455-0450

crossref DOI: https://dx.doi.org/10.18535/jmscr/v8i2.16

\title{
A Rare Case of Retroperitoneal Hydatid Cyst
}

\author{
S. Sravani Kumari ${ }^{1}$, G S Kejriwal ${ }^{2}$, Dr Madhavi $\mathrm{CH}^{3}$, Y.V.R.S. Aparna ${ }^{4}$ \\ ${ }^{1}$ Post Graduate, Dept of Radiology, Maharajah's Institute of Medical Sciences \\ ${ }^{2,3}$ Professor, Dept of Radiology, Maharajah's Institute of Medical Sciences \\ ${ }^{4}$ Senior Resident, Dept of Radiology, Maharajah's Institute of Medical Sciences
}

\begin{abstract}
Introduction: Hydatid disease (HD) is an infestation caused by the larval stage of Echinococcus granulosus. It can be seen in any part of the body, but the liver is the most common site. Retroperitoneal $H D$ is rare, and an isolated retroperitoneal hydatid cyst is extremely rare. Diagnosis of hydatid cyst at unusual locations \& with varied imaging appearances is challenging.

Case Presentation: A 24yrs old lactating female came to gynecology OPD for tubectomy. She was asymptomatic. On examination, palpable mass is noted \& was advised USG abdomen. On USG revealed a large lobulated thick-walled cystic lesion with layered turbid collection within it, in left adnexa, and CECT abdomen is suggestive of retroperitoneal hydatid cyst/ cystic neoplasm. PAIR technique was done for the larger cyst \& the rest of the collection with few small cysts was sent to the pathological examination.

Discussion: Cysts in the peritoneum/retroperitoneum are mainly due to spontaneous or traumatic rupture of concomitant hepatic cysts or surgical inoculation of a hepatic cyst. Total cystectomy was considered as the gold standard treatment \& Albendazole, or praziquantel is indicated for inoperable and disseminated cases. PAIR technique is a nonsurgical option.

Conclusion: In an endemic region of hydatid disease, retroperitoneal hydatid cyst should be considered as one of the differential diagnosis, when one encounters cystic retroperitoneal mass.
\end{abstract}

\section{Introduction}

Hydatid disease is commonly caused by the larval stage of the tapeworm Echinococcus granulosus. The life cycle involves two hosts. The definitive host is usually a dog and rarely some other carnivore. The adult worm resides in the small bowel of the definitive host. Its ova are passed in the feces of the infected animal. Sheep is the usual intermediate host that gets infected by the ingestion of ova by grazing the contaminated grass, and humans get infected by contact with the dog or by ingestion of contaminated water and vegetables. ${ }^{[1]}$ The ovum releases the larva called the oncosphere in the duodenum, which enters the portal circulation by passing through the intestinal wall. Thus, it reaches the liver where it grows into a cyst. Primary Retroperitoneal HD is defined as a zone of hydatidosis occurring in the fatty tissue in the space lying behind the posterior parietal peritoneum without any parasitic foci in other $\operatorname{organs}^{[2]}$. 


\section{Case Presentation}

A 24yrs old female with lactational amenorrhea came to gynecology OPD for tubectomy. There is no h/o pain abdomen or mass per abdomen, no constitutional symptoms. Previously she had regular menstrual cycles. Her bowel \& bladder habits were normal. On examination, per abdomen finding was a Nontender, irregular, ill-defined, firm mass with restricted mobility in left lumbar and iliac regions.

Routine laboratory investigations were normal [Hb- 11.6gms, TC-6,000 cells /cumm, DC (N-66, L-24, E-5 \&M -5\%)]. AEC-300cells/cumm \& CRP-POSITIVE.

Abdominal USG showed a large lobulated thickwalled lesion of size $16 \times 10 \times 8 \mathrm{~cm}$, with layered turbid collection within it, in left adnexa, and extending superiorly upto the level of umbilicus [Figure 1]- likely infected hydatid cyst / cystic mass with the tubo-ovarian originand suggested CECT abdomen and pelvis for further evaluation.

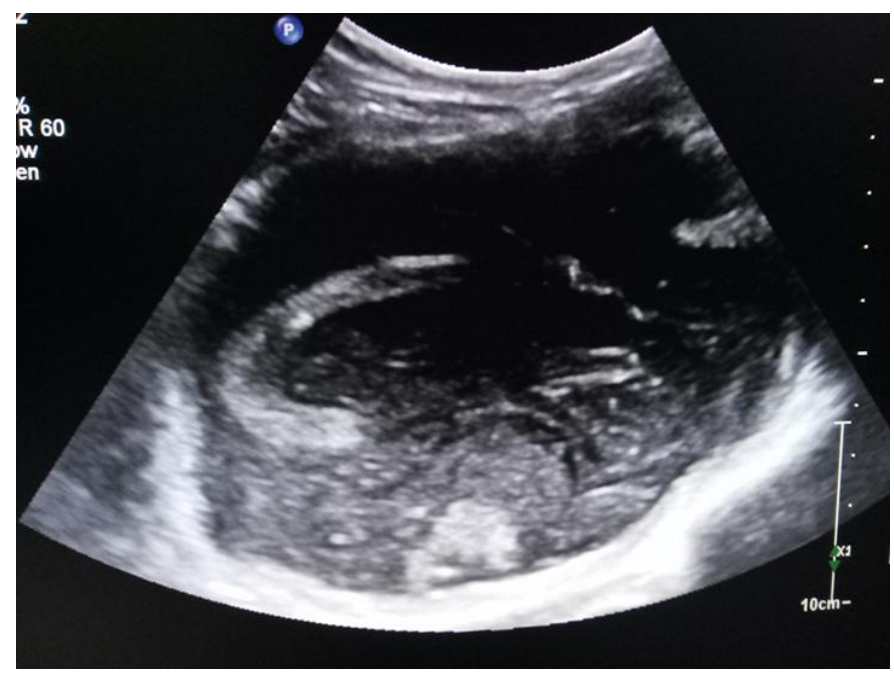

Figure 1: Pelvic collection in an Ultrasound.

CECT abdomen and pelvis revealed a large, welldefined oval-shaped thick-walled multiseptated cystic mass of size $16.1 \times 9.3 \times 9.1 \mathrm{~cm}$, with thin floating membranes in the left iliac \& lumbar regions [Figure 2], extending from the lower pole of left kidney, inferiorly upto the acetabular roof [Figure 3]. Medially the lesion is causing mild right-sided displacement of aorta \& IVC. Laterally, the lesion is abutting the abdominal wall. Descending \& sigmoid colon were displaced anteromedially towards the right side [Figure 3]. On IV contrast administration, there is evidence of minimal enhancement of walls \& septations. The lesion is probably arising from retroperitoneum the diagnostic possibilities are Retroperitoneal hydatid cyst/Cystic neoplasm.

ELISA for echinococcal antigen: IgG 1.30

The patient underwent an exploratory laparotomy. Operative findings were large cystic mass ruptured intraoperatively with two pockets of pus noted in the medial and inferior wall of cyst seen retroperitoneally. Multiple daughter cysts are noted. PAIR technique was done for the larger cyst using $20 \% \mathrm{Nacl}$ as scolicidal agent \& rest of the collection with few small cysts was sent to pathological examination. The abdomen was packed with $10 \%$ hypertonic saline-soaked pads. Pathologic examination of the biopsy material revealed ectocyst consisting of acellular, chitinous laminated hyaline material [Figure $6 \& 7$ ]. Culture of the drainage fluid yielded Klebsiella pneumoniae and short term albendazole treatment was applied together with cefazolin + gentamicin combination against Klebsiella pneumoniae infection. The patient got discharged after a week. A follow-up USG abdomen \& pelvis done after one month following surgery did not reveal any recurrence.

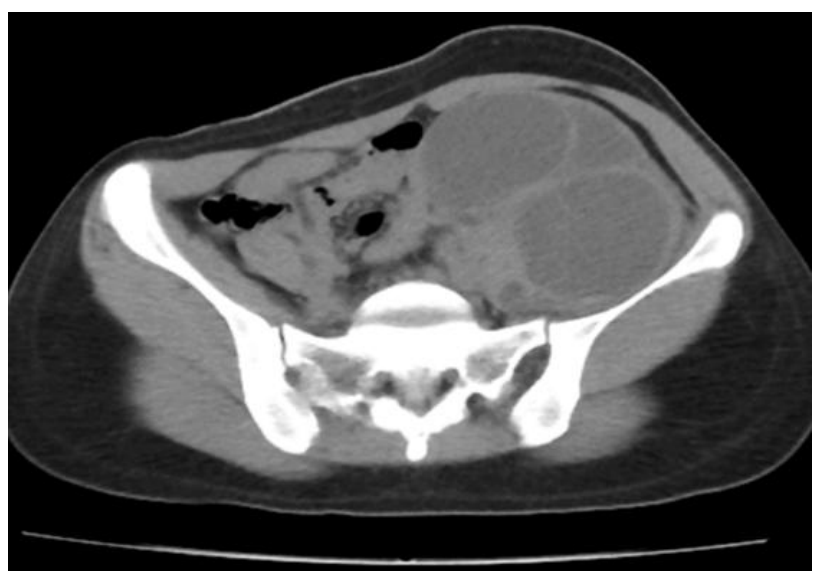

Figure 2: Plain CT Abdomen \& pelvis showing thick walled multiseptated mass with minimal enhancement of walls and septations 


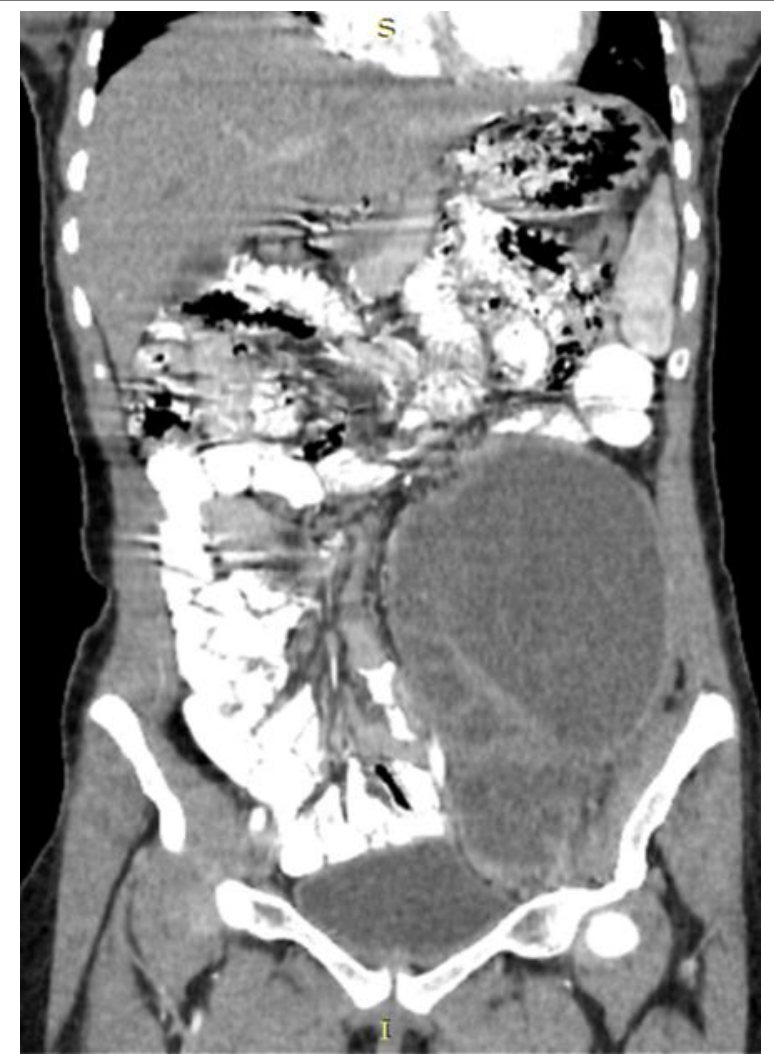

Figure 3: Bowel loops and great vessels are displaced antero-medially towards right side. Laterally, the mass is abutting the abdominal wall.

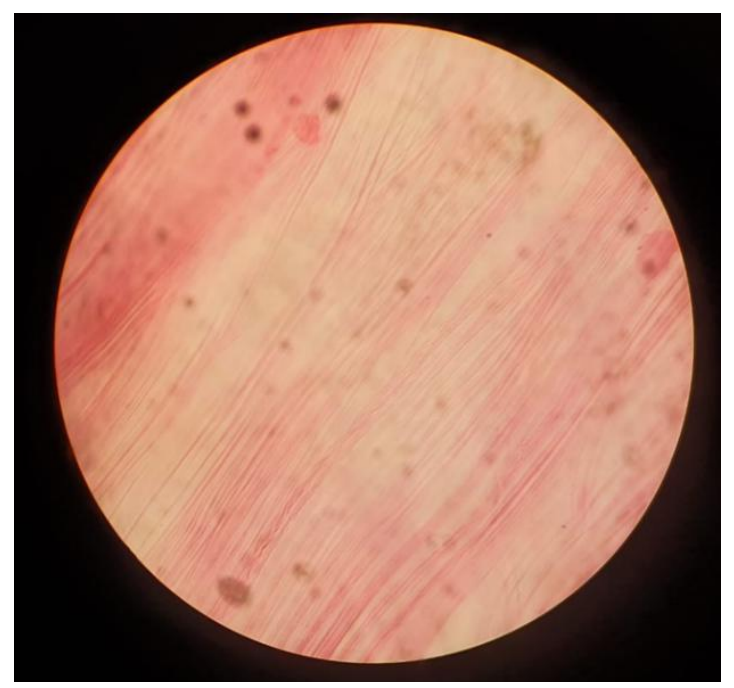

Figure 7: High power field microscopy showing a cellular ectocyst of the hydatid cyst.

\section{Discussion}

The most common cause of an isolated retroperitoneal hydatid cyst could be the hematogenous spread of protoscoleces bypassing the solid organs (liver /lung) or by the gastrointestinal tract into the lymphatic system ${ }^{[3]}$. Diagnosis of a retroperitoneal HD needs a combined assessment of clinical, radiological, and serological analyses (ELISA \& IHAT).

Commonly hydatid cyst has three layers:

1) The outer pericyst consists of modified host cells that form a dense fibrous, protective layer;

2) Middle laminated layer consists of acellular and impervious to bacteria, but allows the passage of nutrients; and

3) The inner germinal layer where the scolices and laminated membrane are produced. The middle laminated layer and the germinal layer form endocyst. ${ }^{[4]}$

US evaluation of hydatid cysts may reveal a welldefined anechoic mass with or without hydatid sand and septa, a dividing septa or daughter cysts within the fluid-filled mass, calcified cysts, and undulating membranes. ${ }^{[5]}$ On CT they may appear as a well-defined hypodense mass with enhancing septa, a cyst with round daughter cysts arranged at the periphery, calcified areas within the cyst, and also undulating membranes. ${ }^{[6]}$ On MRI: Typically, cysts are of low intensity on T1WI and of the high intensity on T2WI. The septa and cyst wall enhance after injection of contrast material, and a low signal intensity rim on T2- weighted MRI is a characteristic finding of hydatidosis. It probably represents pericyst, which is rich in collagen. On T1- and T2-weighted MRI images, daughter cysts manifest as hypointense or isointense areas compared to the maternal matrix ${ }^{[5]}$. Retroperitoneal hydatid usually is seen as an abdominal mass and less frequently with concomitant compression syndromes. It can also rupture into great vessels like the aorta forming false aneurysms ${ }^{[7]}$.

Complications: Complications: Rupture of the cyst \& superinfection. Hepatic hydatid cysts are usually superinfected with varying microorganisms, including E.coli, Enterococcus sp., Staphylococcus aureus, Klebsiella sp., Haemophilus influenza, and streptococci. In extrahepatic hydatid cases, Aspergillus fumigatus, Salmonella sp., and streptococcal infected cysts in the thorax ${ }^{[8]}$. 


\section{Management}

Extrahepatic HD depends on the size, location, and manifestations of the cysts, and the patient's condition. Anti-helminthic drugs are indicated for asymptomatic small cysts ${ }^{[9]}$. Surgical resection is indicated in symptomatic and large cysts as the only curative treatment ${ }^{[9,10]}$. Total cystectomy is considered the gold standard ${ }^{[9]}$. The abdominal cavity should be isolated with gauzes soaked in $20 \%$ hypertonic saline solution for preventing secondary hydatidosis and allergic reactions ${ }^{[9]}$. Scolicidal agents should be used to avoid spillage of the cyst contents. For inoperable and disseminated cases, Albendazole or praziquantel is indicated. Percutaneous aspiration, injection, and re-aspiration (PAIR) technique is a nonsurgical option.

\section{Conclusion}

In an endemic region of hydatid disease, retroperitoneal hydatid cyst must be considered as a differential diagnosis when one encounters multiseptated cystic retroperitoneal mass.Purulent cyst fluid present in all infected cases should be a warning sign for surgeons performing hydatid cyst surgery, and cyst content should be subjected to microbiologic examination in addition to pathological assessment.

\section{References}

1. Beggs I. The radiology of hydatid disease. AJR Am J Roentgenol. 1985; 145:639-48. [PubMed].

2. el-Ouakdi-M, Ben-Fadhel-S, Ayed-M, Zmerli-S Primary Retroperitoneal Hydatid cyst. J-Urol-Paris. 1988; 94 [9-10] 445-8

3. M. R. Erdem, A. Akbaş, F. F. Onol, Y. Tanidir, and S. Y. Onol, "An unusual retroperitoneal sero-negative hydatid cyst presenting with lower urinary tract symptoms," Türkiye Parazitolojii Dergisi, vol. 33, no. 1, pp. 82-84, 2009.View at: Google Scholar.
4. Pedrosa I, Saiz A, Arrozola I, Ferreirós J, Pedrosa CS. Hydatid disease: Radiologic and pathologic features and complications Radiographics. 2000;

20:795-817. [PubMed]

5. P. Polat, M. Kantarci, F. Alper, S. Suma, M.B. Koruyucu, A. Okur Hydatid disease from head to toe.

6. K.J. Gossios, D.S. Kontoyiannis, M. Dasca logiannaki, N.C. Gourtsoyiannis

Uncommon locations of hydatid disease: CT appearances Eur Radiol, 7 (1997), pp. 1303-1308

7. Hadijat-n; Graba-A; Mansouri-H Rupture of retroperitoneal hydatid cyst into the abdominal aorta. Ann-Vasc-Surg 1987 May; 1(4): 483-485

8. Agarwal, R., Ayyagari, A., Yadav, V.B., Prasad, K.N. \& Saraswat, V.A. (2000). Infection of hepatic hydatid cyst with Yersinia enterocolitica. Transactions of the Royal Society of Tropical Medicine and Hygiene 94(4): 404.

9. K. Tepetes, G. Christodoulidis, M. Spryridakis, and K. Hatzitheofilou, "Large solitary retroperitoneal echinococcal cyst: a rare case report," World Journal of Gastroenterology, vol. 13, no. 45, pp. 6101-6103, 2007.

10. G.M. Ettorre, G. Vennarecci, R. Santoro et al., "Giant hydatid cyst of the liver with a retroperitoneal growth: a case report," Journal of Medical Case Reports, vol. 6, no. 1, article 298, 2012. 